\title{
Numerical Analysis on Aerodynamic Characteristics of Delta Wing with Variable Geometry Device in Supersonic Flow
}

\author{
By Masashi KANAMORI ${ }^{1)}$, Osamu IMAMURA ${ }^{1)}$ and Kojiro SUZUKI ${ }^{2)}$ \\ 1) Department of Aeronautics and Astronautics, The University of Tokyo, Tokyo, Japan \\ ${ }^{2)}$ Department of Advanced Energy, The University of Tokyo, Tokyo, Japan
}

(Received April 30th, 2008)

\begin{abstract}
The application of the variable geometry (VG) wing to a lifting re-entry body is expected to enhance the control capability of its aerodynamic characteristics and, as a result, to widen the corridor for the flight trajectory. In the present study, the flow field around a plain delta wing having three chord-wise hinges, one is on the wing root and the others on both sides of the mid-span of the wing, at Mach number 3 is numerically investigated by solving the Euler equations. The effects of the angle of attack and the "tip-down" bending angles around these hinges are clarified. The results show that the lift-to-drag ratio is hardly affected by the tip-down angle and that the overall lift and drag forces vary almost proportional to the change in the projected wing area by taking the tip-down configuration. The center of pressure moves backward by the tip-down effect.
\end{abstract}

Key Words: CFD, Variable Geometry, Delta Wing

$\begin{array}{cll}\text { Nomenclature } & \\ t & : & \text { thickness } \\ x, y, z & : & \text { space coordinate } \\ C_{L} & : & \text { lift coefficient } \\ C_{D} & : & \text { drag coefficient } \\ \delta C_{L} & : & \left(C_{L}\right)_{B}-\left(C_{L}\right)_{0} \\ \delta C_{D} & : & \left(C_{D}\right)_{B}-\left(C_{D}\right)_{0} \\ L / D & : & \text { lift-to-drag ratio } \\ S & : & \text { reference area } \\ \alpha & : & \text { angle of attack } \\ \psi & : & \text { bending angle at center line } \\ \phi & : & \text { bending angle at wing tip } \\ \text { Subscripts } & & \\ 0 & : & \text { result of non-bending wing } \\ B & : & \text { result of bending wing }\end{array}$

\section{Introduction}

It is difficult to design a wing shape which has the aerodynamic characteristics of high efficiency in all the flight regimes ranging from low-speed to supersonic/hypersonic speeds. Variable Geometry (VG) seems to be a promising technology to solve such problems. Some aircrafts with VGwing have been successfully developed so far, for example, $\mathrm{F}-14^{1)}$ and $\mathrm{XB}-70^{2)}$, which have the wings with variable sweep-back angle and unhedral (tip-down) angle, respectively. In the latter case, both sides of the wing tips are bent downward when it flies at supersonic speed.

This "tip-down" device seems to be effective also for the space transportation vehicle of horizontal landing type, because large down-range and cross-range performance can be achieved by high $L / D$ capability of the "tip-down" delta wing in supersonic and hypersonic flight regimes, and lower landing speed can be achieved by setting the wing tips back to the horizontal position and obtaining the maximum plan form area. Such concave configuration of the "tip-down" delta wing is expected to be suitable for riding on the high pressure compressed by shock wave. Moreover, this "tip- down" device is also useful for controlling the pitching motion of the wing. This is explained as the shift of the center of pressure ${ }^{2)}$. Therefore the "tip-down" device is expected to act like elevators.

Many researches on the VG devices have been done so far. For example, the wing with variable cambers ${ }^{3)}$ or variable sweep-back angle ${ }^{4)}$ have been investigated. But there are no researches on the "tip-down" VG device. Therefore, in this research, we deal with this type of the VG device and consider an application of the VG device for a lifting re-entry vehicle as illustrated in Fig.1. In our concept, the vehicle has a plain delta wing with three chord-wise hinges, one is on the wing root and the others on both sides of the mid-span of the wing. The wing should be deployed after the peak heating, because the combination of a plain thin wing and chord-wise hinges cannot survive under the severe aerodynamic heating. The configuration should be as simple as possible. In case of the complicated arrangement of the hinges, the mass required for the VG system will become impractically heavy.

The objective of this study is to investigate the aerodynamic characteristics of a delta wing with VG device and to discuss its application to aerodynamic control, in supersonic flight regime by the computational fluid dynamics (CFD).

\section{Analysis Model and Numerical Methods}

The analysis model is shown in Fig.2. Considering the shape of the XB-70 aircraft ${ }^{2)}$, the flow around a thin delta wing with the sharp leading edge is numerically analyzed. At present, the body is not included in the analyses. In order to simulate the "tip-down" device, various bent angles are considered. Bending down at the wing root is also considered. Bending angles at the wing root and tips are 0,10 and 20 degrees. The typical computational grid around the model is shown in Fig.3. The number of the grid points are $110 \times$ $201 \times 60$ in the chord-wise, the span-wise, and the direction away from the wing surface, respectively. The convergence of the lift coefficient with respect to the number of the grid points has been investigated. The error of the lift coefficient 


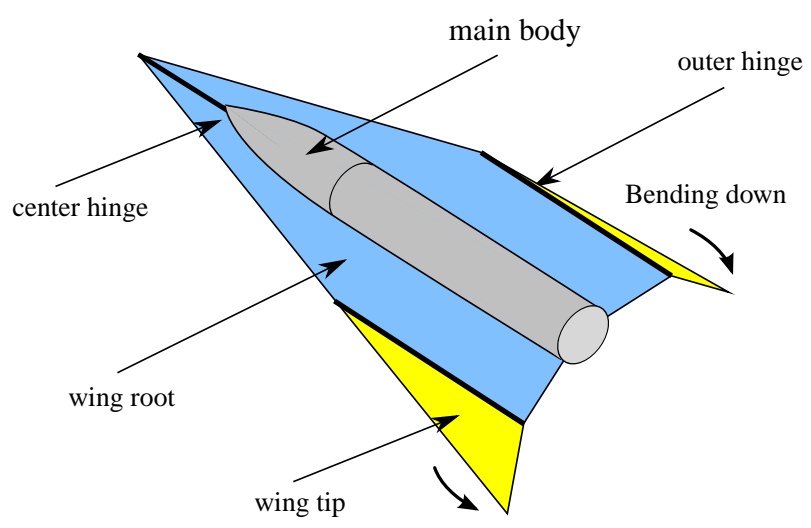

Fig.1 Concept of lifting re-entry vehicle with VG wing having chord-wise hinges

between this grid and finer one was within $1 \%$.

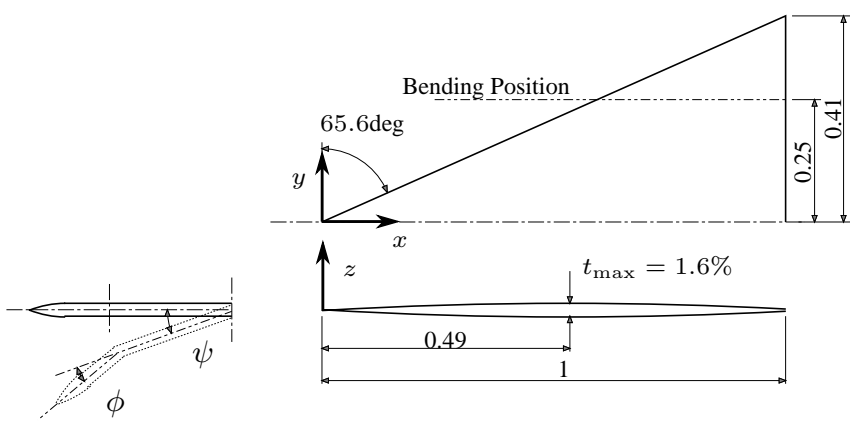

Fig.2 Three views of analysis model (nondimensionalized by the chord length)

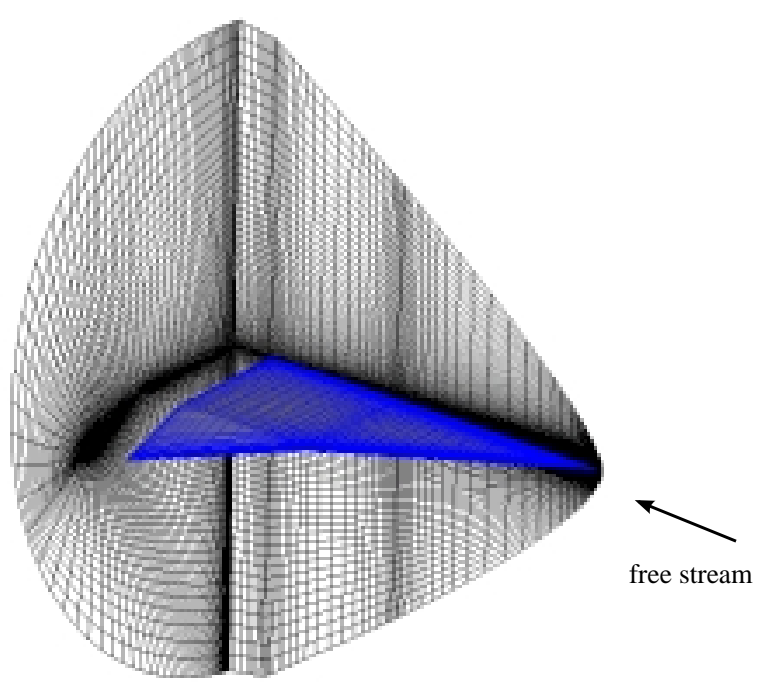

Fig.3 Typical computational grid around the model

In the present study, the wing is quite thin and the effect of viscosity is significant only in the thin boundary layer over the surface. The angle of attack is small in the present calculation and no separation occurs in the flow field around the wing. We focus on the effects of the VG device on the aerodynamic characteristics of the vehicle. Therefore, the three-dimensional compressible Euler equations are used as the governing equations for the flow calculation. The SHUS scheme ${ }^{5)}$ with 3rd-order MUSCL interpolation ${ }^{6)}$ is used for calculating the numerical inviscid flux. The LU-SGS implicit scheme ${ }^{7)}$ is applied for time integration, because we only consider the steady state solutions. The computation has been conducted at the free stream Mach number 3 and the angles of attack 0, 2 and 4 degrees.

\section{Result and Discussion}

Figure 4 shows the variations of $C_{L}, C_{D}$ and $L / D$ with the angle of attack for various wing root and tip bent angles. In Fig.4, $C_{L}, C_{D}$ and $L / D$ are defined as follows:

$$
\begin{aligned}
C_{L} & =\frac{L}{\frac{1}{2} \rho_{\infty} U_{\infty}^{2} S} \\
C_{D} & =\frac{D}{\frac{1}{2} \rho_{\infty} U_{\infty}^{2} S} \\
L / D & =\frac{C_{L}}{C_{D}}=\frac{L}{D}
\end{aligned}
$$

where $S$ denotes the reference area of the wing. The reference area is defined as a plan form area projected to the horizontal plane including the center hinge (see Fig.1). It should be noted that the reference area decreases when the bending angle increases. As seen in Fig.4, the aerodynamic coefficients seem hardly affected by the wing bending. This fact means that both the drag and the lift forces decrease with the increase in the bending angle.

The patterns of the wing bending are classified into three types as illustrated in Fig.5. For convenience of the explanation, these are called as Case 1, 2 and 3. In Case 1, only the effect of wing tips bending is considered. Wing root bending is considered in Case 2. Case 3 is defined as the combination of these 2 cases. In other words, both the wing root and the wing tips are bent in Case 3. In order to evaluate precisely the effects of the wing bending, the difference in $C_{L}$ and $C_{D}$ from the result of the nonbending case are calculated and plotted in Fig.6 and 7, respectively. Considering from Fig.6, the increments of the lift coefficient are positive in all cases. But result of Case 3 is less than the sum of that of Case 1 and 2, although Case 3 is a combination of Case 1 and 2 . For example, $\delta C_{L}$ at $\psi=10 \mathrm{deg}, \phi=10 \mathrm{deg}$ is less than the sum of $\delta C_{L}$ at $\psi=0 \mathrm{deg}, \phi=10 \mathrm{deg}$ and that at $\psi=10 \mathrm{deg}, \phi=0 \mathrm{deg}$. By contrast, the increments of the drag coefficient, which are shown in Fig.7, show different characters from that of the lift coefficient. In some results, namely, Case 1 with $\psi=0 \mathrm{deg}, \phi=10 \mathrm{deg}$, Case 2 with $\psi=10 \mathrm{deg}, \phi=0 \mathrm{deg}$, and Case 3 with $\psi=10 \mathrm{deg}, \phi=10 \mathrm{deg}, \delta C_{D}$ in Case 3 is the smallest of those in Case 1 and 2. Moreover, $\delta C_{D}$ becomes negative in some results of Case 3. This means that the drag force is reduced by bending the wing in the tip-down direction.

The chord-wise distributions of the lift and drag forces are shown in Fig.8 and 9, respectively. Definition of the coordinate system is shown in Fig.2. The lift force is augmented in 

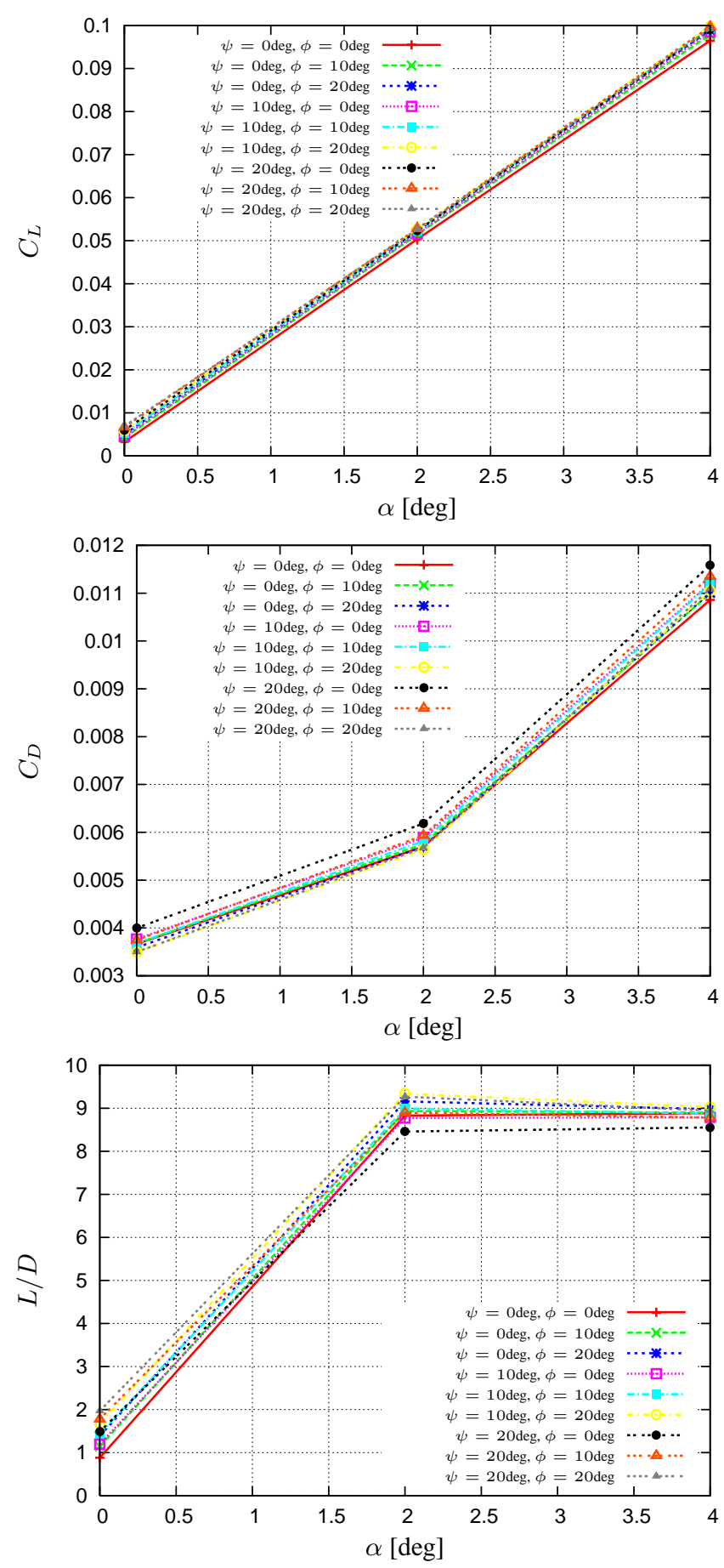

Fig.4 Aerodynamic coefficients for various bent angles $\underline{\text { Case } 1}$

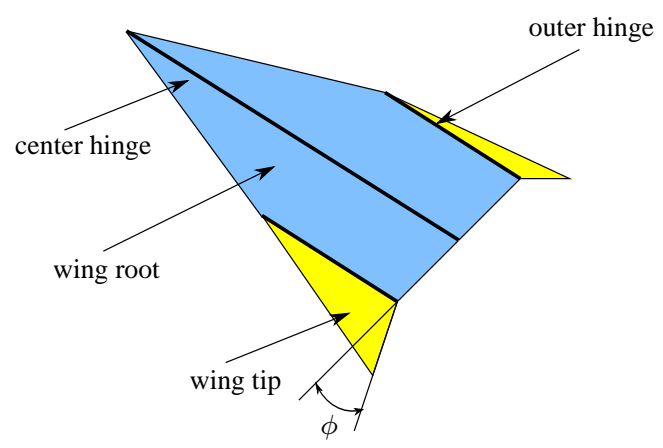

Case 2

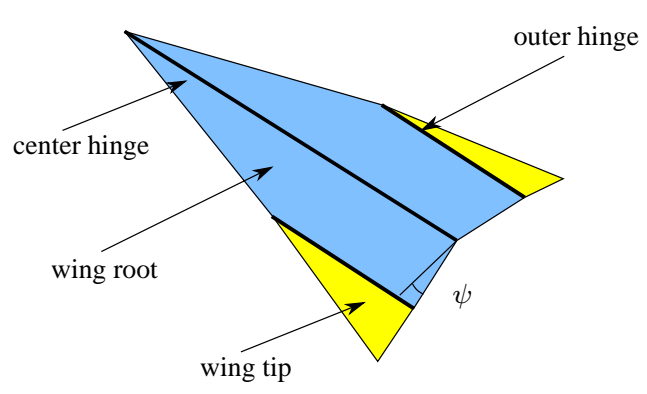

$\underline{\text { Case } 3}$

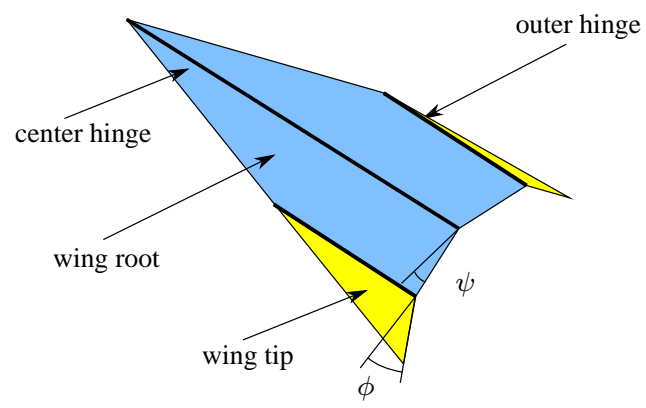

Fig.5 Classification of wing bending cases

the region from the bending point (about $60 \%$ root chord) to the trailing edge in Case 1 . In Case 2, augmentation of the lift distribution is observed at every chord position. The results of Case 3, however, are not the superposition of that of Case 1 and Case 2. In fact, the result at $\psi=20 \mathrm{deg}, \phi=20 \mathrm{deg}$ in Case 3 shows decrease of $C_{L}$ in the region from $80 \%$ root chord to the trailing edge. On the other hand, each of distribution of the drag is almost the same.

The contour lines of the pressure coefficient on the plane at $70 \%$ root chord are shown in Fig.10. Shock wave can be confirmed at the lower side of the wing in each case. All the cases have almost the same values of the maximum pressure. This causes that $C_{L}$ and $C_{D}$ have almost the same values with respect to the bending of the wing.

Finally, the effect of the wing bending on the location of the center of pressure is discussed, which is illustrated in Fig.11. In Fig.11, M.A.C. in the label of the vertical axis stands for the mean aerodynamic chord, which is defined as 


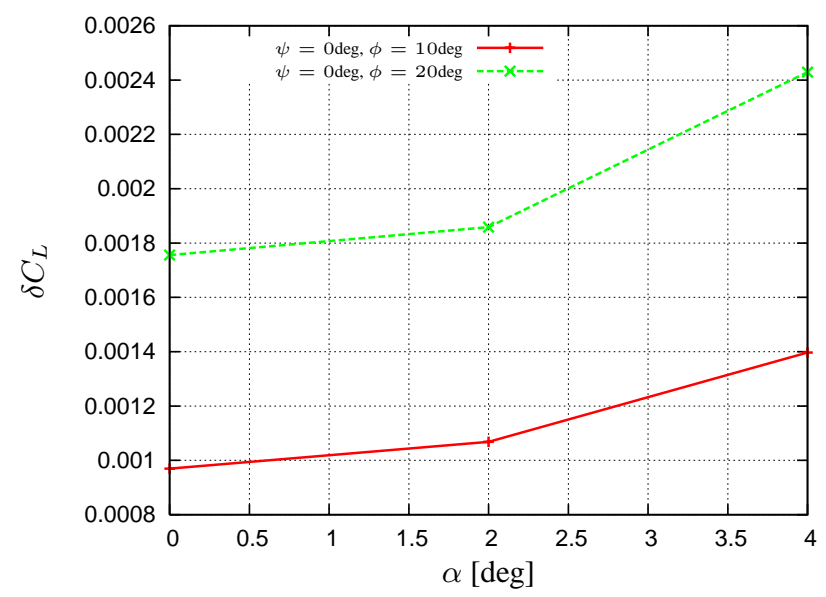

Case 1

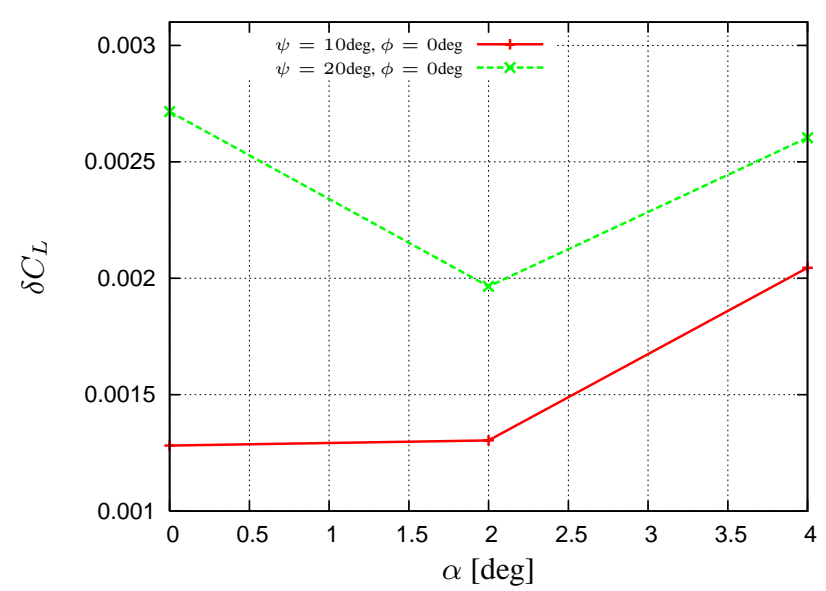

Case 2

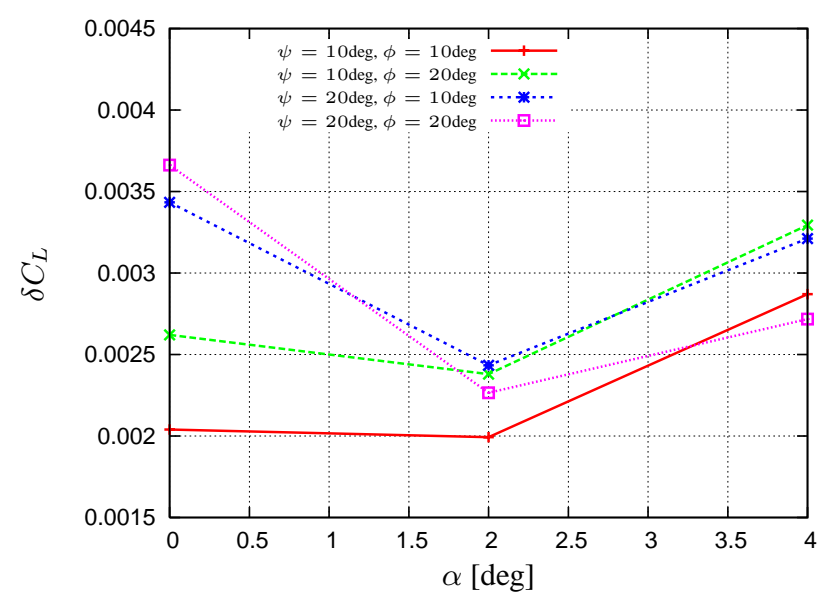

Case 3

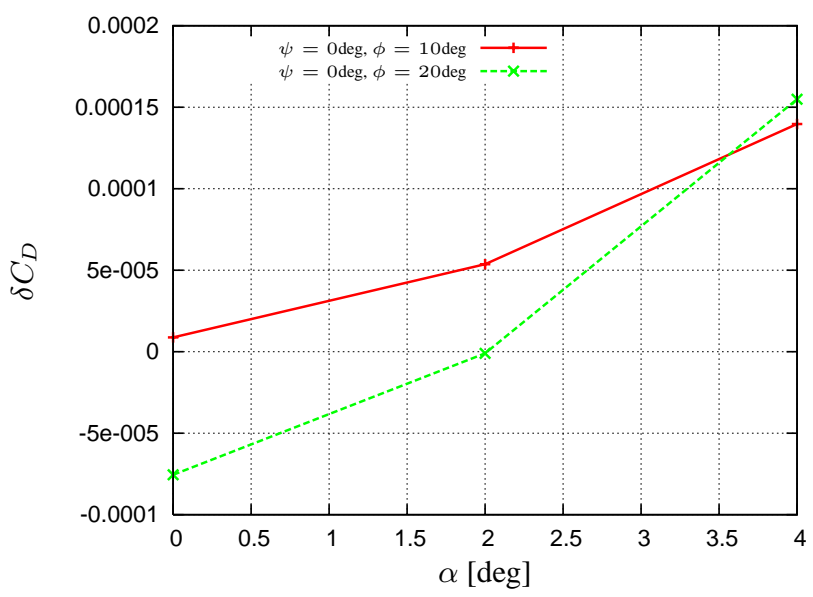

Case 1

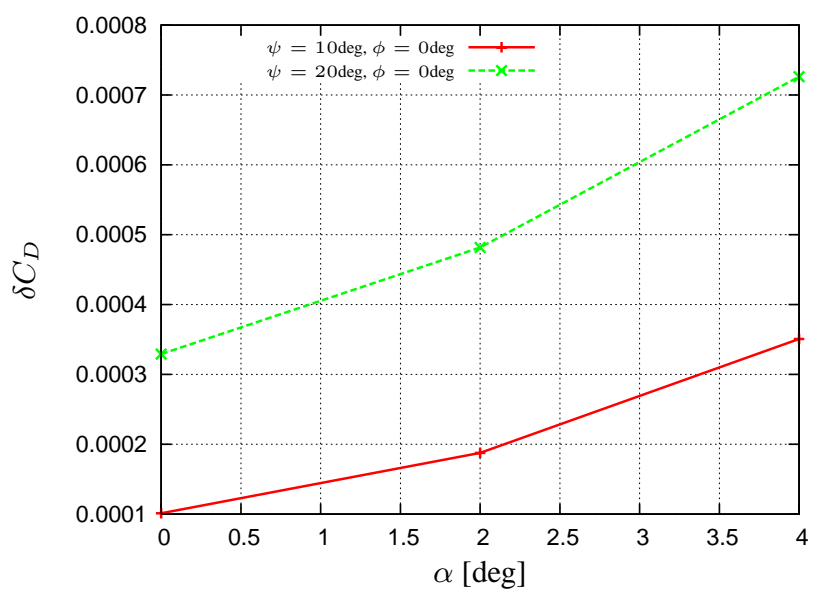

Case 2

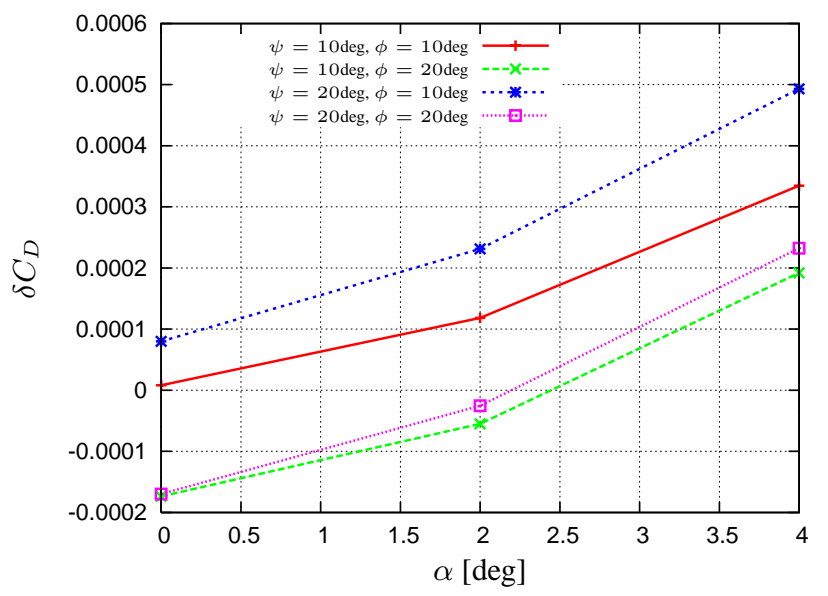

Case 3

Fig.7 Changes of drag coefficient due to bending 


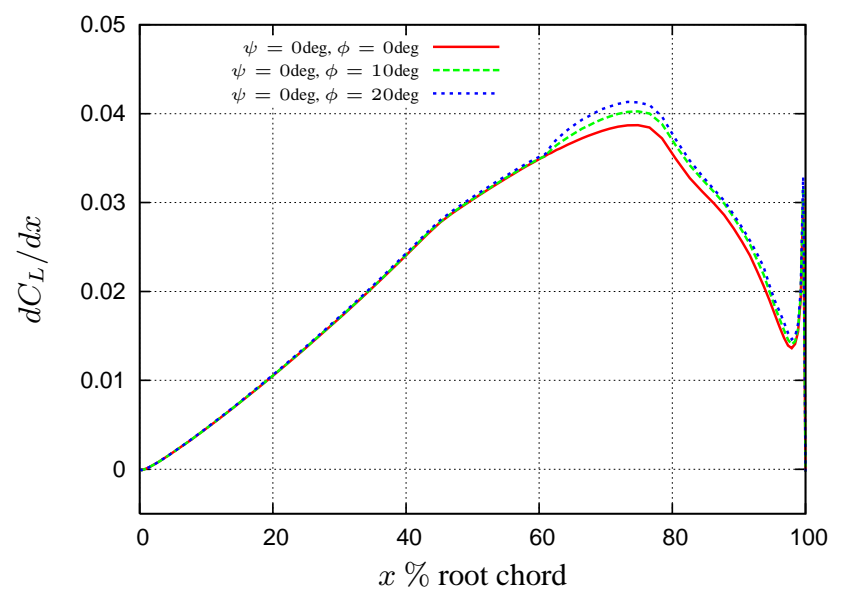

Case 1

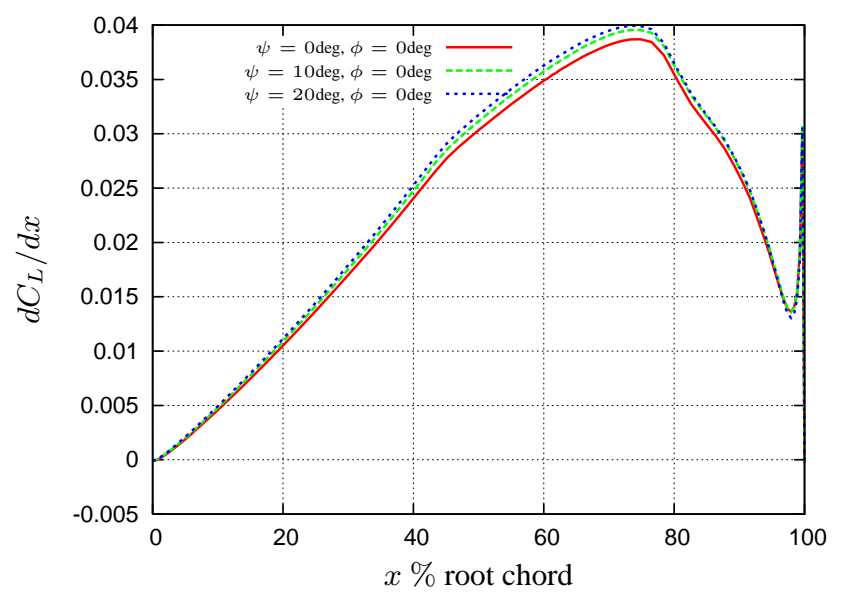

Case 2

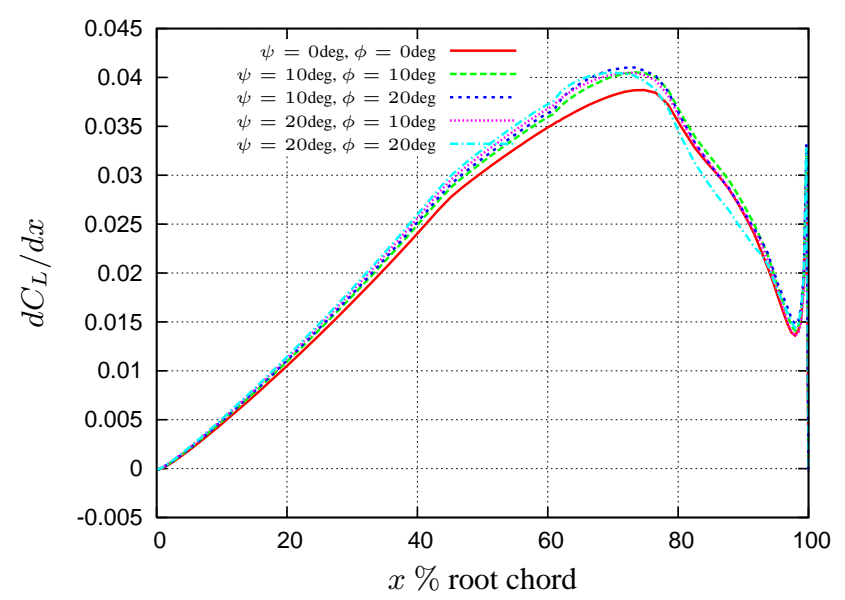

Case 3

Fig.8 Chord-wise distribution of lift coefficient $(\alpha=2 \mathrm{deg})$

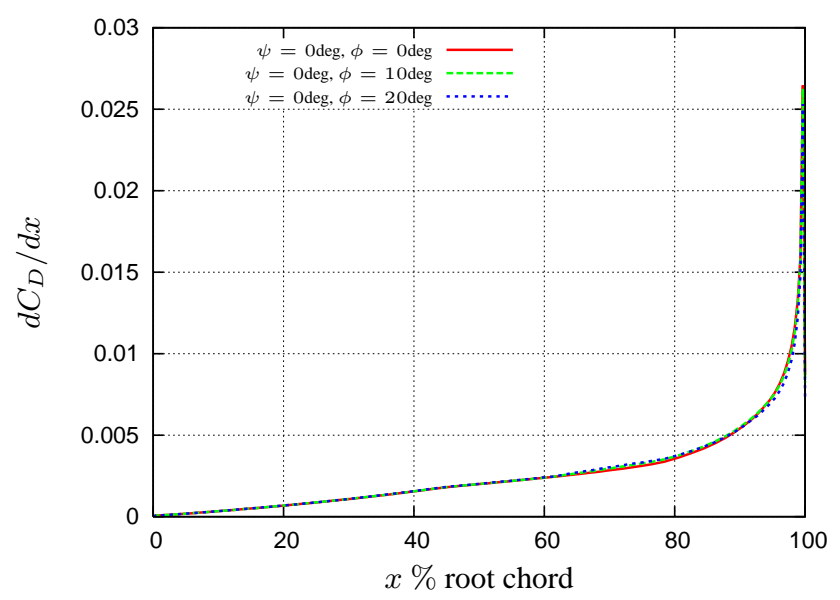

Case 1

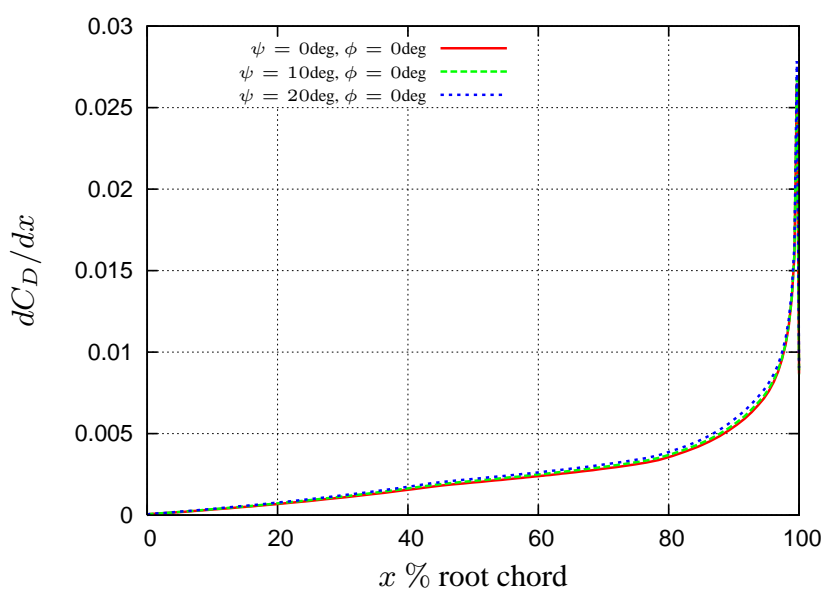

Case 2

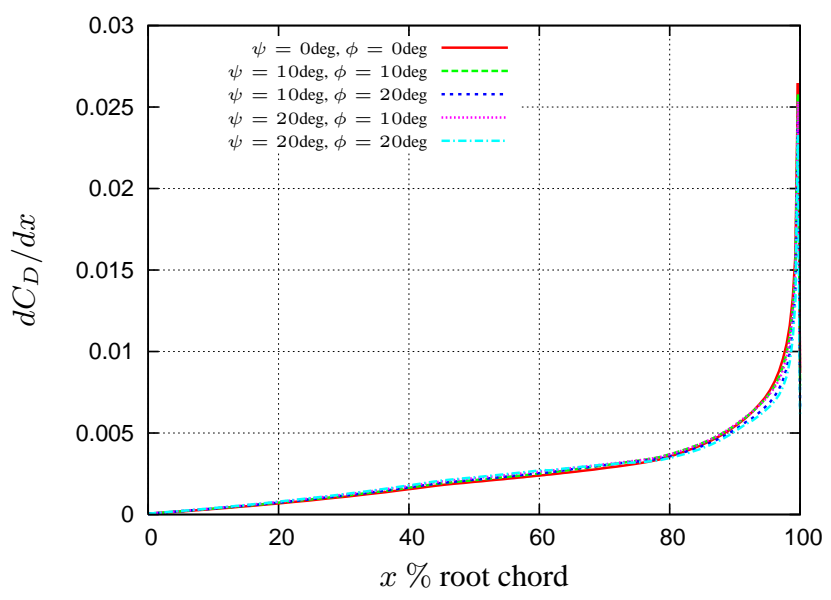

Case 3

Fig.9 Chord-wise distribution of drag coefficient $(\alpha=2 \mathrm{deg})$ 

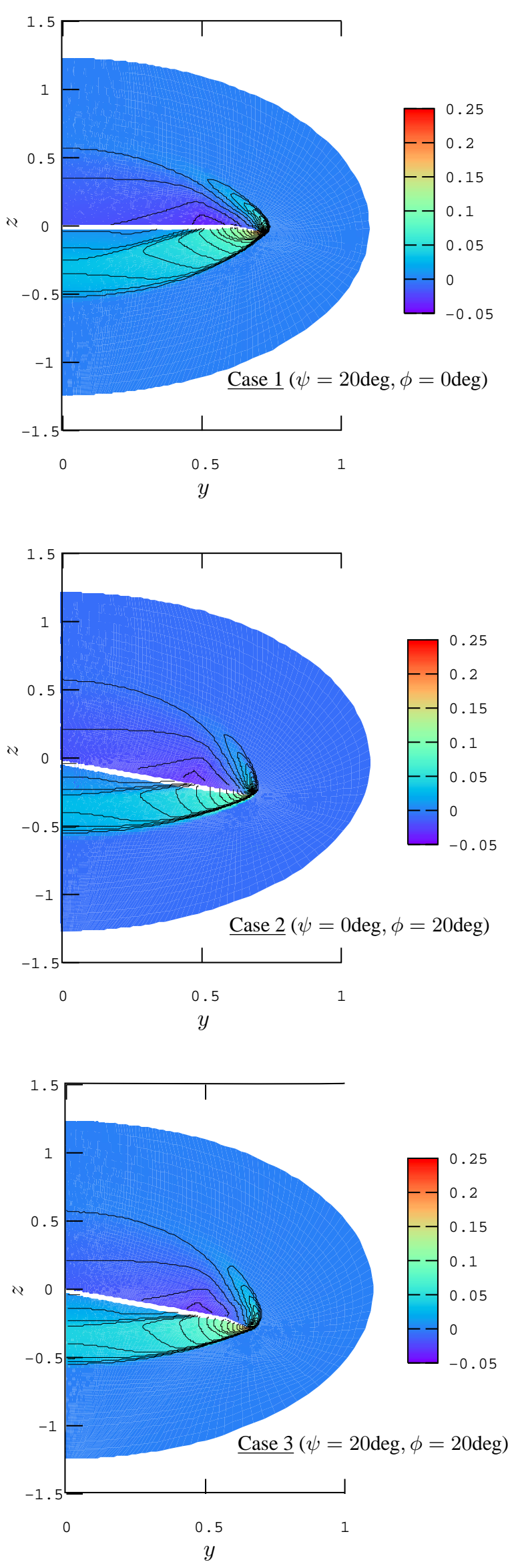

Fig.10 Contour lines of pressure coefficient at $70 \%$ root chord for various bent angles a chord at one-third of the span from the center line. Figure 11 shows that the center of pressure shifts backward in all cases of bending. This shift can be explained as follows: by bending the wing, the center of drag force shifts downward compared to that for non-bending. This shift means that the moment arm from the center of drag forces to the origin of the coordinate system $O$ becomes farther, as illustrated in Fig.12. On the other hand, lift and drag forces are hardly affected by the bent angle. Therefore, total pitching moment in a head-down direction increases, because the pitching moment due to drag increases and that due to lift hardly changes. As a result, the center of pressure shifts backward so that the resultant force can be balanced with the pitching moment.

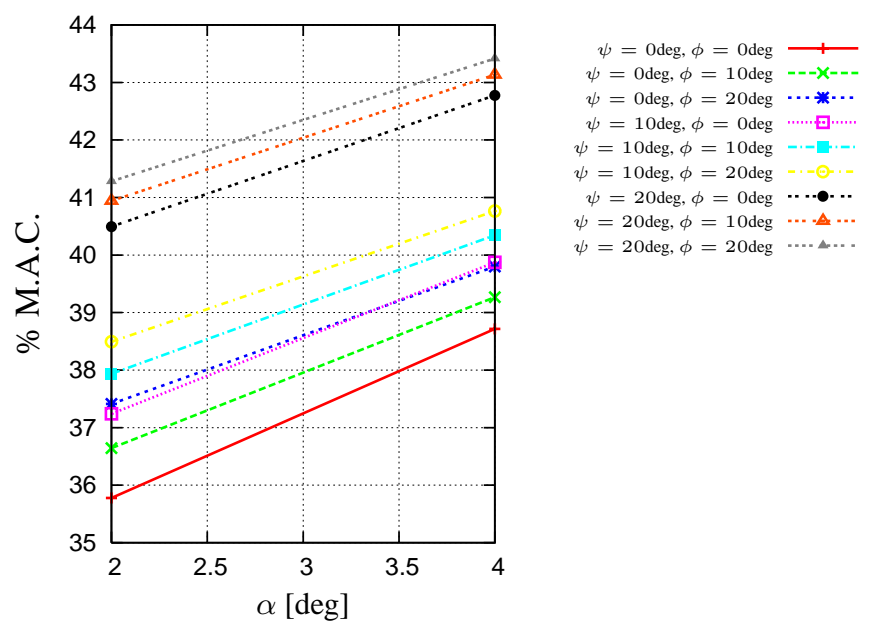

Fig.11 Shift of the center of pressure due to wing bending

This means that there are some application possibilities to control the pitching motion of the wing. Elevators is generally used to control the pitching motion of the aircraft. But operating this device causes the reductions of the lift and drag coefficients. On the other hand, the wing with "tipdown" devices can control its pitching motion without any reductions of the aerodynamic coefficients. Therefore, bending the wing is more useful for controlling the pitching motion than operating the elevators.

\section{Concluding Remarks}

Aerodynamic characteristics of VG devices are investigated by the CFD. All the aerodynamic coefficients are hardly affected by the wing bending. Minor augmentation in $L / D$ is confirmed in the result. This is mainly due to the decrease of the drag rather than the increase of the lift. The center of pressure is also affected by wing bending and it shifts backward. This means that there are some application possibilities to control the pitching motion of the wing. This study shows that the effect of the wing bending is useful for applying to the control of the pitching motion rather than modifying the lift and drag coefficients.

\section{References}

1) Munson, K. Jackson, P. and Peacock, L.: Jane's all the world's aircraft, Janes Information Group, 2008. 

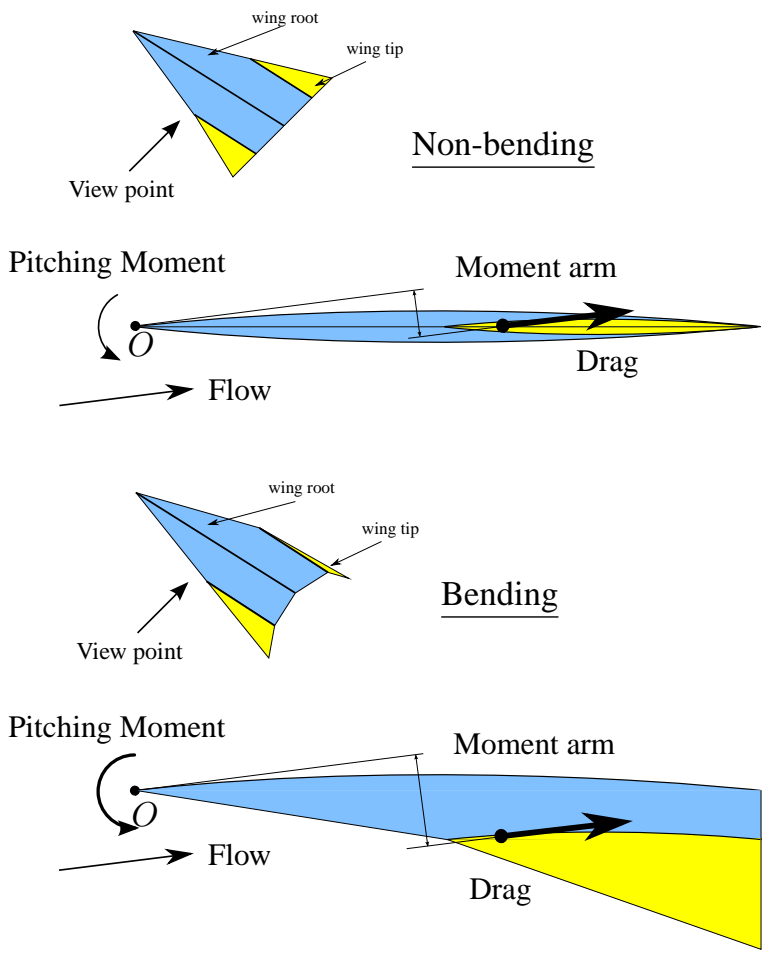

Fig.12 Decrease of pitching moment due to the shift of the center of drag
2) Campbell, J. M.: North American XB-70 Valkyrie: The Legacy (Schiffer Military History Book), Schiffer Pub Ltd, 1998.

3) Gilyard, G. B. and Bolonkin, A.: Estimated benefits of variablegeometry wing camber control for transport aircraft, NASA/TM-1999206586, (1999).

4) Beissner, F. L. Jr., Domack, C. S., Shields, E. W. and Martin, G. L.: The influence of subsonic mission segments on the use of variable-sweep wings for high speed civil transport configurations, AIAA Paper, 884470, (1988).

5) Shima, E and Jounouchi, T.: Role of CFD in aeronautical engineering (No.14) - AUSM type upwind schemes, Proc. 14th NAL Symp. Aircraft Comput. Aerodyn., (1999), pp. 7-24 .

6) van Leer, B.: Toward the ultimate conservative difference scheme. 4 a new approach to numerical convection, J. Comput. Phys., 23 (1977), pp. 276-299.

7) Yoon, D. and Kwak, S.: An implicit three-dimensional Navier-Stokes solver for compressible flow, AIAA J., 30 (1992), pp. 2635-2659. 